Coch, F.: Wespenbekämpfung in Lebensmittelverkaufsstellen. Angewandte Parasitologie. 11, 1970. S. 225 bis 231 .

Insbesondere in Bäckereien, Konditoreien, aber auch in anderen Verkaufsstellen von Lebensmitteln wie auch in Lebensmittelbetrieben treten fast alljährlich in den Sommermonaten Juni bis September, auch Anfang Oktober Wespen u. zw. Paravespula germanica und $P$. vulgaris auf. Es werden zwei Bekämpfungsarten beschrieben, die den Wespenbefall in den betreffenden Objekten innerhalb von ein bis fünf Wochen beseitigen. Die eine Methode besteht in Besprühen der Schaufensterrahmen, der Fensterbretter und der Schaufenster selbst, dieser in einer Breite von $50 \mathrm{~cm}$ vom Rande her. Die Behandlung erfolgt mit mindertoxischen Pyrethrumpräparaten. Am geeignetsten erwies sich ein Delitia-Py-Druckzerstäuber und eine Nebula Spritze mit dem Pyrethrum-Wespenpräparat der Firma Delitia. Die Behandlung selbst erfolgte zur Zeit, wo sich im Raume keinerlei Lebensmittel befanden. Als zweite erfolgreiche Methode wurde eine UV-Lichtfalle angewendet. Diese hat eine Leistungsaufnahme von 40 Watt, eine Drehzahl von 200 U/Min., eine Spannung von $220 \mathrm{~V} / 5 \mathrm{~Hz}$, Spezialleuchtstofflampe von UV-A; mittlere Wellenlänge $335 \mathrm{~nm}$. Die Wirkung beruht auf der Attraktivität des ultravioletten Lichtes für fliegende Insekten. Das Gerät hat einen Wirkungsbereich von $85 \mathrm{~m}^{2}$. Für beide Verfahren ist das Verhalten der Wespen, nach dem Abflug vom Futterplatz sich dem Licht zuzuwenden, ausschlaggebend. Der Fallenmethode ist aus toxikologischen Gründen der Vorzug zu geben. Die dem UV-Licht zustrebenden Insekten werden durch den Sog des Ventilators in den Beutel geschleudert, der mit einem Insektizid imprägniert wird. Regelmäßig so durchgeführte Bekämpfungen dürften die am Zuflug beteiligten Nestgemeinschaften ausschalten.

\section{E. Sch.}

\section{INSEKTIZIDE}

Das DDT-Dossier - Eine kritische Besprechung.

Henri Sirrez, Diplomlandwirt und Ministerialrat im französischen Landwirtschaftsministerium, hat in der Zeitschrift Phytoma-Defense des Cultures eine Artikelserie mit dem Titel DDT-Dossier herausgegeben, die in einer 44seitigen bebilderten Broschüre zusammengefaßt worden ist; aus dieser Zusammenfassung ergeben sich folgende Tatsachen:

1969 wurden in der Welt noch ungefähr 90000 Tonnen des DDT-haltigen Insektizids hergestellt. Diese Produktion, die seit mehreren Jahren rückläufig ist, verringert sich auch im Jahre 1970, und zwar um etwa $20 \%$. Die Hälfte wird von der Weltgesundheitsorganisation (WHO) insbesondere für die Malariabekämpfung verwendet, $3 / 4$ der restlichen Menge dienen dem Schutz von Baumwollanpflanzungen. Für die übrigen landwirtschaftlichen Verwendungszwecke verblieben danach nur eine relativ geringe DDT-Menge. Diese Schlußfolgerung bedarf einer Uberprüfung.

Der erste Teil der von SIRIEz 1969 fertiggestellten Studie befaßt sich mit der Herstellung und Verwendung von DDT sowie mit den Vor- und Nachteilen dieses Insektizids. Kapitel 2 und 3 sind mit "DDT und Gesundheit des Menschen" und "DDT und Fauna" betitelt. Der Verfasser analysiert verschiedene Daten und bestimmte Fakten. Das Ergebnis dieser Analyse bedarf noch einer kritischen Betrachtung.

Im vierten Kapitel werden nähere Angaben über die Einschränkungen beim Gebrauch von DDT in den USA und in Europa gemadht. Allgemeine Verwendungsverbote, von denen häufig berichtet worden ist, werden nur in Einzelfällen registriert. In Schweden hat die Forstverwaltung für die Bekämpfung des Kiefernrüsselkäfers eine Ausnahmegenehmigung für 13 Tonnen erhalten, die mehr als $1 / 5$ der früher in diesem Land verwendeten Gesamtmenge entspricht.

In Frankreich wurde das DDT gleichzeitig mit dem HCH nur für die Stallbehandlung verboten. Die Verwendungseinschränkungen in der Bundesrepublik
Deutschland werden ein größeres Ausmaß erreichen. Sie werden - mit Ausnahme eines engen Anwendungsbereichs im Forst - einem generellen Verbot gleichkommen. Eine entsprechende Verordnung ist anfangs des Jahres 1971 zu erwarten. Im übrigen ist. bisher weder in den sechs EWG-Ländern noch in Großbritannien ein generelles DDT-Verbot ausgesprochen worden; in diesen Ländern hat sich die Verwendung von DDT auf Grund der Entwicklung anderer nichtpersistenter Insektizide seit einem Jahrzehnt beträchtlich verringert.

In den Vereinigten Staaten haben fünf - von $50-$ Bundestaaten, in denen keine Baumwolle angepflanzt wird und die in ihrer Landwirtschaft sehr wenig DDT verwendeten, den Gebrauch grundsätzlich untersagt. Auf Bundesebene hat das US-Landwirtschaftsministerium am 20. November 1969 die Streichung der "Eintragung" von DDT für die vier folgenden Verwendungszwecke beschlossen: 1. Behandlung von Zierbäumen; 2. Behandlung von Tabakpflanzungen; 3. Verwendung in Haus und Garten; 4. Jede Verwendung in Wasserwirtschafts- und Sumpfgebieten; diese vier Verwendungszwecke machen $35 \%$ des in den USA sowohl für die Landwirtschaft als auch für die Volksgesundheit verwendeten DDT aus. Einige DDT-Hersteller haben gegen diese Entscheidung Berufung eingelegt. Im übrigen hat der "Ausschuß für den Umweltschutz" der unmiftelbar Präsident NrxoN untersteht, vorgeschlagen, das DDT in allen Fällen, in denen es durch andere weniger persistente Insektizide ersetzt werden könnte, zu verbieten. Dieser Vorschlag entspricht im übrigen einer FAO-Empfehlung und den Gründen für die seit einigen Jahren rückläufige DDT-Produktion. Demzufolge sind 1970 in den USA neue Einschränkungen für andere Verwendungszwecke als für Baumwollpflanzungen ausgespochen worden. Ahnlichen Überlegungen entspringen auch die für die Bundesrepublik Deutschland vorgesehenen Verbote und Einschränkungen.

$$
-\mathrm{Lb}-
$$

Heinrich, E., H. Beitz, H. Reifenstein, J. Hartisch, F. Seefeld und M. Dunsing (1970): Pflanzenschutzmittel-Rückstände im Boden als Ursache für unbeabsichtigte Sekundärwirkungen. Nachrichtenblatt f. d. Deutschen Pflanzenschutzdienst, S. 251-256.

In der vorliegenden Arbeit wird das wichtige Problem der Rückstände von Pflanzenschutzmitteln und zwar von Insektiziden, Akariziden, Herbiziden und Fungiziden behandelt. Die gröBte Bedeutung bei der Kontamination des Bodens haben die Insektizide und von diesen die persistenten Chlorkohlenwasserstoffe. Bei früheren umfangreichen Untersuchungen konnten die Autoren bei DDT einen hohen Kontaminationsgrad auf landwirtschaftlich und gärtnerisch genutzten Böden der Deutschen Demokratischen Republik nachweisen. Ein günstigeres Bild zeigte das Verhalten von Lindane im Boden, dies ist deshalb von Interesse, da diesem Wirkstoff mit dem stufenweisen Ersatz des DDT immer mehr Bedeutung zukommt. Die von Hernisch und seinen Mitarbeitern untersuchten, vorwiegend landwirtschaftlich genutzten Flächen bestätigen die günstige Wirkung von Lindane. Herbizide, die in der DDR angewendet werden, insbesondere Verbindungen aus der Gruppe der s-Triazine, Harnstoffderivate, sowie das Chloralhydrat und Chlorat, verfügen über eine beträchtliche Persistenz in verschiedenen Böden bei verschiedenen ökologischen Bedingungen. Dadurch können Folgekulturen geschädigt und die Fruchtbarkeit des Bodens herabgesetzt werden. In dieser Tabelle werden einige Beispiele zur Presistenz von Simazain, vor allem nach Behandlung mit AtrazinPräparaten angeführt. Die Fungizide weisen im Boden - von wenigen Ausnahmen abgesehen - eine geringe Persistenz auf. Es interessieren vor allem die Organo-Quecksilberverbindungen (zur Saatgutbeizung) sowie die Polychlornitrobenzole.

A. Sinreich 